\title{
Measurement of the $Z Z$ production cross section and search for the standard model Higgs boson in the four lepton final state in $p \bar{p}$ collisions
}

V. M. Abazov, ${ }^{31}$ B. Abbott, ${ }^{66}$ B. S. Acharya,${ }^{25}$ M. Adams,${ }^{45}$ T. Adams, ${ }^{43}$ J. P. Agnew, ${ }^{40}$ G. D. Alexeev, ${ }^{31}$ G. Alkhazov, ${ }^{35}$ A. Alton, ${ }^{55}{ }^{*}$ A. Askew, ${ }^{43}$ S. Atkins,${ }^{53}$ K. Augsten, ${ }^{7}$ C. Avila,${ }^{5}$ F. Badaud,${ }^{10}$ L. Bagby, ${ }^{44}$ B. Baldin, ${ }^{44}$ D. V. Bandurin, ${ }^{43}$ S. Banerjee, ${ }^{25}$ E. Barberis,${ }^{54}$ P. Baringer, ${ }^{52}$ J. F. Bartlett, ${ }^{44}$ U. Bassler, ${ }^{15}$ V. Bazterra, ${ }^{45}$ A. Bean,${ }^{52}$ M. Begalli, ${ }^{2}$ L. Bellantoni, ${ }^{44}$ S. B. Beri, ${ }^{23}$ G. Bernardi, ${ }^{14}$ R. Bernhard, ${ }^{19}$ I. Bertram ${ }^{38}$ M. Besançon, ${ }^{15}$ R. Beuselinck, ${ }^{39}$ P. C. Bhat, ${ }^{44}$ S. Bhatia, ${ }^{57}$ V. Bhatnagar, ${ }^{23}$ G. Blazey, ${ }^{46}$ S. Blessing, ${ }^{43}$ K. Bloom, ${ }^{58}$ A. Boehnlein, ${ }^{44}$ D. Boline,${ }^{63}$ E. E. Boos,${ }^{33}$ G. Borissov, ${ }^{38}$ A. Brandt, ${ }^{69}$ O. Brandt, ${ }^{20}$ R. Brock,${ }^{56}$ A. Bross, ${ }^{44}$ D. Brown, ${ }^{14}$ X. B. Bu, ${ }^{44}$ M. Buehler, ${ }^{44}$ V. Buescher,${ }^{21}$ V. Bunichev, ${ }^{33}$ S. Burdin, ${ }^{38,}$ C. P. Buszello, ${ }^{37}$ E. Camacho-Pérez,${ }^{28}$ B. C. K. Casey, ${ }^{44}$ H. Castilla-Valdez, ${ }^{28}$ S. Caughron, ${ }^{56}$ S. Chakrabarti, ${ }^{63}$ K. M. Chan, ${ }^{50}$ A. Chandra ${ }^{71}$ E. Chapon, ${ }^{15}$ G. Chen, ${ }^{52}$ S. W. Cho, ${ }^{27}$ S. Choi, ${ }^{27}$ B. Choudhary, ${ }^{24}$ S. Cihangir, ${ }^{44}$ D. Claes,${ }^{58}$ J. Clutter, ${ }^{52}$ M. Cooke, ${ }^{44}$ W. E. Cooper, ${ }^{44}$ M. Corcoran, ${ }^{71}$ F. Couderc, ${ }^{15}$ M.-C. Cousinou, ${ }^{12}$ D. Cutts, ${ }^{68}$ A. Das,${ }^{41}$ G. Davies, ${ }^{39}$ S. J. de Jong, ${ }^{29,30}$ E. De La Cruz-Burelo, ${ }^{28}$ F. Déliot,${ }^{15}$ R. Demina,${ }^{62}$ D. Denisov, ${ }^{44}$ S. P. Denisov, ${ }^{34}$ S. Desai, ${ }^{44}$ C. Deterre,${ }^{20,}$ K. DeVaughan,${ }^{58}$ H. T. Diehl, ${ }^{44}$ M. Diesburg,${ }^{44}$ P. F. Ding, ${ }^{40}$ A. Dominguez,${ }^{58}$ A. Dubey,${ }^{24}$ L. V. Dudko, ${ }^{33}$ A. Duperrin, ${ }^{12}$ S. Dutt,${ }^{23}$ M. Eads,${ }^{46}$ D. Edmunds,${ }^{56}$ J. Ellison, ${ }^{42}$ V. D. Elvira, ${ }^{44}$ Y. Enari, ${ }^{14}$ H. Evans,${ }^{48}$ V. N. Evdokimov,${ }^{34}$ L. Feng, ${ }^{46}$ T. Ferbel, ${ }^{62}$ F. Fiedler, ${ }^{21}$ F. Filthaut, ${ }^{29,30}$ W. Fisher, ${ }^{56}$ H. E. Fisk,${ }^{44}$ M. Fortner, ${ }^{46}$

H. Fox, ${ }^{38}$ S. Fuess, ${ }^{44}$ A. Garcia-Bellido, ${ }^{62}$ J. A. García-González, ${ }^{28}$ V. Gavrilov, ${ }^{32}$ W. Geng, ${ }^{12,56}$ C. E. Gerber, ${ }^{45}$ Y. Gershtein, ${ }^{59}$ G. Ginther, ${ }^{44,62}$ G. Golovanov, ${ }^{31}$ P. D. Grannis, ${ }^{63}$ S. Greder, ${ }^{16}$ H. Greenlee, ${ }^{44}$ G. Grenier, ${ }^{17}$ Ph. Gris, ${ }^{10}$ J.-F. Grivaz, ${ }^{13}$ A. Grohsjean, ${ }^{15, \$}$ S. Grünendahl, ${ }^{44}$ M. W. Grünewald, ${ }^{26}$ T. Guillemin, ${ }^{13}$ G. Gutierrez, ${ }^{44}$ P. Gutierrez, ${ }^{66}$ J. Haley, ${ }^{54}$ L. Han, ${ }^{4}$ K. Harder, ${ }^{40}$ A. Harel, ${ }^{62}$ J. M. Hauptman, ${ }^{51}$ J. Hays, ${ }^{39}$ T. Head,${ }^{40}$ T. Hebbeker, ${ }^{18}$ D. Hedin,${ }^{46}$

H. Hegab,${ }^{67}$ A. P. Heinson, ${ }^{42}$ U. Heintz,${ }^{68}$ C. Hensel,${ }^{20}$ I. Heredia-De La Cruz ${ }^{28,8}$ K. Herner,${ }^{44}$ G. Hesketh,${ }^{40, \pi}$ M. D. Hildreth ${ }^{50}$ R. Hirosky, ${ }^{72}$ T. Hoang, ${ }^{43}$ J. D. Hobbs, ${ }^{63}$ B. Hoeneisen, ${ }^{9}$ J. Hogan, ${ }^{71}$ M. Hohlfeld, ${ }^{21}$ R. Hooper,,${ }^{68,\|\|}$ I. Howley, ${ }^{69}$ Z. Hubacek, ${ }^{7,15}$ V. Hynek, ${ }^{7}$ I. Iashvili, ${ }^{61}$ Y. Ilchenko, ${ }^{70}$ R. Illingworth, ${ }^{44}$ A. S. Ito, ${ }^{44}$ S. Jabeen, ${ }^{68}$ M. Jaffré, ${ }^{13}$ A. Jayasinghe, ${ }^{66}$ J. Holzbauer, ${ }^{57}$ M. S. Jeong, ${ }^{27}$ R. Jesik, ${ }^{39}$ P. Jiang, ${ }^{4}$ K. Johns, ${ }^{41}$ E. Johnson, ${ }^{56}$ M. Johnson, ${ }^{44}$ A. Jonckheere, ${ }^{44}$ P. Jonsson, ${ }^{39}$ J. Joshi, ${ }^{42}$ A. W. Jung, ${ }^{44}$ A. Juste, ${ }^{36}$ E. Kajfasz, ${ }^{12}$ D. Karmanov, ${ }^{33}$ I. Katsanos, ${ }^{58}$ R. Kehoe, ${ }^{70}$ S. Kermiche, ${ }^{12}$ N. Khalatyan, ${ }^{44}$ A. Khanov, ${ }^{67}$ A. Kharchilava, ${ }^{61}$ Y. N. Kharzheev, ${ }^{31}$ I. Kiselevich,${ }^{32}$ J. M. Kohli, ${ }^{23}$

A. V. Kozelov, ${ }^{34}$ J. Kraus,${ }^{57}$ A. Kumar,${ }^{61}$ A. Kupco, ${ }^{8}$ T. Kurča,${ }^{17}$ V. A. Kuzmin ${ }^{33}$ S. Lammers,${ }^{48}$ P. Lebrun, ${ }^{17}$ H. S. Lee, ${ }^{27}$ S. W. Lee, ${ }^{51}$ W. M. Lee, ${ }^{43}$ X. Lei, ${ }^{41}$ J. Lellouch, ${ }^{14}$ D. Li, ${ }^{14}$ H. Li, ${ }^{72}$ L. Li, ${ }^{42}$ Q. Z. Li, ${ }^{44}$ J. K. Lim, ${ }^{27}$ D. Lincoln, ${ }^{44}$ J. Linnemann, ${ }^{56}$ V. V. Lipaev, ${ }^{34}$ R. Lipton, ${ }^{44}$ H. Liu ${ }^{70}$ Y. Liu, ${ }^{4}$ A. Lobodenko, ${ }^{35}$ M. Lokajicek, ${ }^{8}$ R. Lopes de Sa, ${ }^{63}$ R. Luna-Garcia, ${ }^{28, * *}$ A. L. Lyon, ${ }^{44}$ A. K. A. Maciel, ${ }^{1}$ R. Madar, ${ }^{19}$ R. Magaña-Villalba, ${ }^{28}$ S. Malik, ${ }^{58}$ V. L. Malyshev,${ }^{31}$ J. Mansour, ${ }^{20}$ J. Martínez-Ortega ${ }^{28}$ R. McCarthy, ${ }^{63}$ C. L. McGivern,${ }^{40}$ M. M. Meijer, ${ }^{29,74}$ A. Melnitchouk,${ }^{44}$ D. Menezes,${ }^{46}$ P. G. Mercadante, ${ }^{3}$ M. Merkin, ${ }^{33}$ A. Meyer, ${ }^{18}$ J. Meyer,${ }^{20,}{ }^{+1}$ F. Miconi, ${ }^{16}$ N. K. Mondal,${ }^{25}$ M. Mulhearn, ${ }^{72}$ E. Nagy, ${ }^{12}$ M. Narain, ${ }^{68}$ R. Nayyar, ${ }^{41}$ H. A. Neal, ${ }^{55}$ J. P. Negret, ${ }^{5}$ P. Neustroev, ${ }^{35}$ H. T. Nguyen, ${ }^{72}$ T. Nunnemann, ${ }^{22}$ J. Orduna,${ }^{71}$ N. Osman, ${ }^{12}$ J. Osta, ${ }^{50}$ A. Pal, ${ }^{69}$ N. Parashar, ${ }^{49}$ V. Parihar, ${ }^{68}$ S. K. Park,${ }^{27}$ R. Partridge, ${ }^{68, \|}$ N. Parua, ${ }^{48}$ A. Patwa, ${ }^{64,8 \S}$ B. Penning, ${ }^{44}$ M. Perfilov, ${ }^{33}$ Y. Peters, ${ }^{20}$ K. Petridis, ${ }^{40}$ G. Petrillo, ${ }^{62}$ P. Pétroff,,${ }^{13}$ M.-A. Pleier, ${ }^{64}$ V. M. Podstavkov, ${ }^{44}$ A. V. Popov, ${ }^{34}$ M. Prewitt, ${ }^{71}$ D. Price, ${ }^{48}$ N. Prokopenko, ${ }^{34}$ J. Qian, ${ }^{55}$ A. Quadt, ${ }^{20}$ B. Quinn,,${ }^{57}$ P. N. Ratoff, ${ }^{38}$ I. Razumov, ${ }^{34}$

I. Ripp-Baudot, ${ }^{16}$ F. Rizatdinova,${ }^{67}$ M. Rominsky, ${ }^{44}$ A. Ross, ${ }^{38}$ C. Royon,${ }^{15}$ P. Rubinov,${ }^{44}$ R. Ruchti, ${ }^{50}$ G. Sajot, ${ }^{11}$ A. Sánchez-Hernández, ${ }^{28}$ M. P. Sanders, ${ }^{22}$ A. S. Santos,${ }^{1, \dagger}$ G. Savage, ${ }^{44}$ L. Sawyer, ${ }^{53}$ T. Scanlon, ${ }^{39}$ R. D. Schamberger ${ }^{63}$ Y. Scheglov, ${ }^{35}$ H. Schellman, ${ }^{47}$ C. Schwanenberger ${ }^{40}$ R. Schwienhorst, ${ }^{56}$ J. Sekaric,${ }^{52}$ H. Severini, ${ }^{66}$ E. Shabalina, ${ }^{20}$ V. Shary, ${ }^{15}$ S. Shaw ${ }^{56}$ A. A. Shchukin, ${ }^{34}$ V. Simak, ${ }^{7}$ P. Skubic, ${ }^{66}$ P. Slattery, ${ }^{62}$ D. Smirnov, ${ }^{50}$ G. R. Snow, ${ }^{58}$ J. Snow, ${ }^{65}$ S. Snyder ${ }^{64}$ S. Söldner-Rembold, ${ }^{40}$ L. Sonnenschein, ${ }^{18}$ K. Soustruznik, ${ }^{6}$ J. Stark, ${ }^{11}$ D. A. Stoyanova, ${ }^{34}$ M. Strauss, ${ }^{66}$ L. Suter, ${ }^{40}$ P. Svoisky, ${ }^{66}$ M. Titov, ${ }^{15}$ V. V. Tokmenin, ${ }^{31}$ Y.-T. Tsai, ${ }^{62}$ D. Tsybychev, ${ }^{63}$ B. Tuchming, ${ }^{15}$ C. Tully, ${ }^{60}$ L. Uvarov ${ }^{35}$ S. Uvarov, ${ }^{35}$ S. Uzunyan, ${ }^{46}$ R. Van Kooten ${ }^{48}$ W. M. van Leeuwen, ${ }^{75}$ N. Varelas, ${ }^{45}$ E. W. Varnes, ${ }^{41}$ I. A. Vasilyev, ${ }^{34}$ A. Y. Verkheev, ${ }^{31}$ L. S. Vertogradov, ${ }^{31}$ M. Verzocchi, ${ }^{44}$ M. Vesterinen, ${ }^{40}$ D. Vilanova, ${ }^{15}$ P. Vokac, ${ }^{7}$ H. D. Wahl,${ }^{43}$ M. H. L. S. Wang, ${ }^{44}$ J. Warchol,${ }^{50}$ G. Watts,${ }^{73}$ M. Wayne, ${ }^{50}$ J. Weichert, ${ }^{21}$ L. Welty-Rieger, ${ }^{47}$ M. R. J. Williams, ${ }^{48}$ G. W. Wilson, ${ }^{52}$ M. Wobisch, ${ }^{53}$ D. R. Wood, ${ }^{54}$ T. R. Wyatt, ${ }^{40}$ Y. Xie, ${ }^{44}$ R. Yamada, ${ }^{44}$ S. Yang, ${ }^{4}$

T. Yasuda, ${ }^{44}$ Y. A. Yatsunenko, ${ }^{31}$ W. Ye, ${ }^{63}$ Z. Ye,${ }^{44}$ H. Yin,${ }^{44}$ K. Yip,${ }^{64}$ S. W. Youn, ${ }^{44}$ J. M. Yu, ${ }^{55}$ J. Zennamo, ${ }^{61}$ T. G. Zhao, ${ }^{40}$ B. Zhou, ${ }^{55}$ J. Zhu, ${ }^{55}$ M. Zielinski, ${ }^{62}$ D. Zieminska, ${ }^{48}$ and L. Zivkovic ${ }^{14}$ 
${ }^{1}$ LAFEX, Centro Brasileiro de Pesquisas Físicas, Rio de Janeiro, Brazil

${ }^{2}$ Universidade do Estado do Rio de Janeiro, Rio de Janeiro, Brazil

${ }^{3}$ Universidade Federal do ABC, Santo André, Brazil

${ }^{4}$ University of Science and Technology of China, Hefei, People's Republic of China

${ }^{5}$ Universidad de los Andes, Bogotá, Colombia

${ }^{6}$ Charles University, Faculty of Mathematics and Physics, Center for Particle Physics, Prague, Czech Republic

${ }^{7}$ Czech Technical University in Prague, Prague, Czech Republic

${ }^{8}$ Institute of Physics, Academy of Sciences of the Czech Republic, Prague, Czech Republic

${ }^{9}$ Universidad San Francisco de Quito, Quito, Ecuador

${ }^{10}$ LPC, Université Blaise Pascal, CNRS/IN2P3, Clermont, France

${ }^{11}$ LPSC, Université Joseph Fourier Grenoble 1, CNRS/IN2P3, Institut National Polytechnique de Grenoble, Grenoble, France

${ }^{12}$ CPPM, Aix-Marseille Université, CNRS/IN2P3, Marseille, France

${ }^{13}$ LAL, Université Paris-Sud, CNRS/IN2P3, Orsay, France

${ }^{14}$ LPNHE, Universités Paris VI and VII, CNRS/IN2P3, Paris, France

${ }^{15}$ CEA, Irfu, SPP, Saclay, France

${ }^{16} I P H C$, Université de Strasbourg, CNRS/IN2P3, Strasbourg, France

${ }^{17}$ IPNL, Université Lyon 1, CNRS/IN2P3, Villeurbanne, France and Université de Lyon, Lyon, France

${ }^{18}$ III. Physikalisches Institut A, RWTH Aachen University, Aachen, Germany

${ }^{19}$ Physikalisches Institut, Universität Freiburg, Freiburg, Germany

${ }^{20}$ II. Physikalisches Institut, Georg-August-Universität Göttingen, Göttingen, Germany

${ }^{21}$ Institut für Physik, Universität Mainz, Mainz, Germany

${ }^{22}$ Ludwig-Maximilians-Universität München, München, Germany

${ }^{23}$ Panjab University, Chandigarh, India

${ }^{24}$ Delhi University, Delhi, India

${ }^{25}$ Tata Institute of Fundamental Research, Mumbai, India

${ }^{26}$ University College Dublin, Dublin, Ireland

${ }^{27}$ Korea Detector Laboratory, Korea University, Seoul, Korea

${ }^{28}$ CINVESTAV, Mexico City, Mexico

${ }^{29}$ Nikhef, Science Park, Amsterdam, The Netherlands

${ }^{30}$ Radboud University Nijmegen, Nijmegen, The Netherlands

${ }^{31}$ Joint Institute for Nuclear Research, Dubna, Russia

${ }^{32}$ Institute for Theoretical and Experimental Physics, Moscow, Russia

${ }^{33}$ Moscow State University, Moscow, Russia

${ }^{34}$ Institute for High Energy Physics, Protvino, Russia

${ }^{35}$ Petersburg Nuclear Physics Institute, St. Petersburg, Russia

${ }^{36}$ Institució Catalana de Recerca i Estudis Avançats (ICREA) and Institut de Física d'Altes Energies (IFAE), Barcelona, Spain

${ }^{37}$ Uppsala University, Uppsala, Sweden

${ }^{38}$ Lancaster University, Lancaster LA1 4YB, United Kingdom

${ }^{39}$ Imperial College London, London SW7 2AZ, United Kingdom

${ }^{40}$ The University of Manchester, Manchester M13 9PL, United Kingdom

${ }^{41}$ University of Arizona, Tucson, Arizona 85721, USA

${ }^{42}$ University of California Riverside, Riverside, California 92521, USA

${ }^{43}$ Florida State University, Tallahassee, Florida 32306, USA

${ }^{44}$ Fermi National Accelerator Laboratory, Batavia, Illinois 60510, USA

${ }^{45}$ University of Illinois at Chicago, Chicago, Illinois 60607, USA

${ }^{46}$ Northern Illinois University, DeKalb, Illinois 60115, USA

${ }^{47}$ Northwestern University, Evanston, Illinois 60208, USA

${ }^{48}$ Indiana University, Bloomington, Indiana 47405, USA

${ }^{49}$ Purdue University Calumet, Hammond, Indiana 46323, USA

${ }^{50}$ University of Notre Dame, Notre Dame, Indiana 46556, USA

${ }^{51}$ Iowa State University, Ames, Iowa 50011, USA

${ }^{52}$ University of Kansas, Lawrence, Kansas 66045, USA

${ }^{53}$ Louisiana Tech University, Ruston, Louisiana 71272, USA

${ }^{54}$ Northeastern University, Boston, Massachusetts 02115, USA

${ }^{55}$ University of Michigan, Ann Arbor, Michigan 48109, USA

${ }^{56}$ Michigan State University, East Lansing, Michigan 48824, USA

${ }^{57}$ University of Mississippi, University, Mississippi 38677, USA

${ }^{58}$ University of Nebraska, Lincoln, Nebraska 68588, USA

${ }^{59}$ Rutgers University, Piscataway, New Jersey 08855, USA

${ }^{60}$ Princeton University, Princeton, New Jersey 08544, USA

${ }^{61}$ State University of New York, Buffalo, New York 14260, USA 


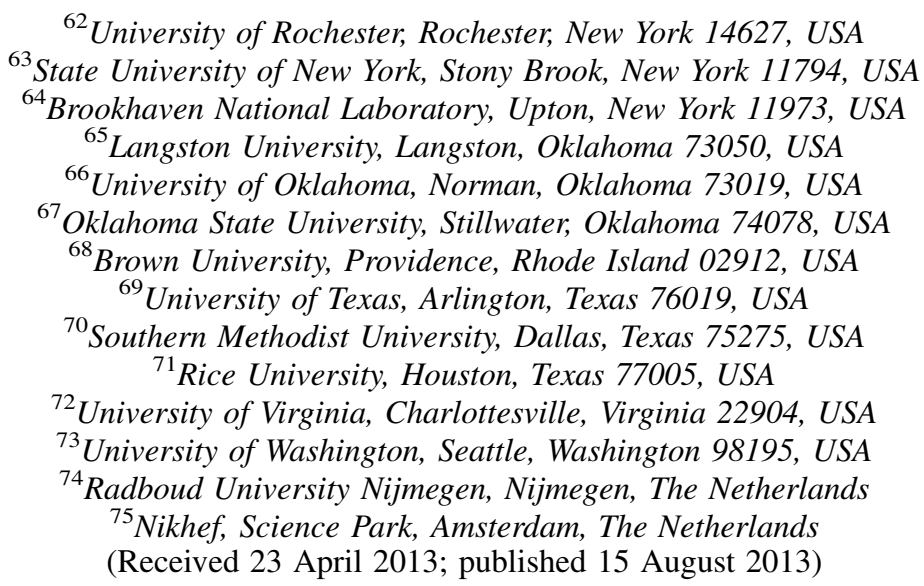

We present a measurement of $Z$ boson pair production in $p \bar{p}$ collisions at $1.96 \mathrm{TeV}$ with 9.6 to $9.8 \mathrm{fb}^{-1}$ of D0 data. We examine the final states eеeе, ее $\mu \mu$, and $\mu \mu \mu \mu$. Based on selected data, the measured cross section in the mass region $M\left(Z / \gamma^{*}\right)>30 \mathrm{GeV}$ is $\sigma\left(p \bar{p} \rightarrow Z / \gamma^{*} Z / \gamma^{*}\right)=1.26_{-0.36}^{+0.44}(\text { stat })_{-0.15}^{+0.17} \times$ (syst) \pm 0.08 (lumi) $\mathrm{pb}$; after correcting for the expected ratio of $\sigma\left(p \bar{p} \rightarrow Z / \gamma^{*} Z / \gamma^{*}\right)$ to $\sigma(p \bar{p} \rightarrow Z Z)$, we derive a cross section for $p \bar{p} \rightarrow Z Z$ production of $1.05_{-0.30}^{+0.37}$ (stat) ${ }_{-0.12}^{+0.14}$ (syst) \pm 0.06 (lumi) pb. This result is combined with a previous result from the $Z Z \rightarrow \ell^{+} \ell^{-} \nu \bar{\nu}$ channel resulting in a combined $p \bar{p} \rightarrow$ $Z Z$ cross section measurement of $1.32_{-0.25}^{+0.29}$ (stat) \pm 0.12 (syst) \pm 0.04 (lumi) pb. These measurements are consistent with the standard model expectation of $1.43 \pm 0.10 \mathrm{pb}$. We extend this analysis to search for the standard model (SM) Higgs boson between 115 and $200 \mathrm{GeV}$. At a Higgs boson mass of $125 \mathrm{GeV}$, we expect to set a limit of 43 times the SM expectation at 95\% C.L., and set a limit of 42 times the SM expectation at $95 \%$ C.L.

DOI: 10.1103/PhysRevD.88.032008

\section{INTRODUCTION}

We present a measurement of the cross section $\sigma\left(p \bar{p} \rightarrow Z / \gamma^{*} Z / \gamma^{*}\right)$ at $\sqrt{s}=1.96 \mathrm{TeV}$, using events where each $Z / \gamma^{*}$ results in two charged leptons. Because the branching fraction of the $Z$ boson to charged leptons is smaller than that to quarks or neutrinos, this process is relatively rare, but has the advantage of being an extremely pure final state. The largest fraction of the background results from events in which one or more jet has been

\footnotetext{
*Visitor from Augustana College, Sioux Falls, South Dakota, USA.

${ }^{\dagger}$ Visitor from The University of Liverpool, Liverpool, United Kingdom.

Visitor from DESY, Hamburg, Germany.

${ }^{\S}$ Visitor from Universidad Michoacana de San Nicolas de Hidalgo, Morelia, Mexico.

"Visitor from SLAC, Menlo Park, California, USA.

${ }^{\text {II } V i s i t o r}$ from University College London, London, United Kingdom.

**Visitor from Centro de Investigacion en Computacion - IPN, Mexico City, Mexico.

${ }^{\dagger}$ Visitor from Universidade Estadual Paulista, São Paulo, Brazil.

\$Visitor from Karlsruher Institut für Technologie (KIT) Steinbuch Centre for Computing (SCC).

${ }_{\S \S}$ Visitor from Office of Science, U.S. Department of Energy, Washington, D.C. 20585, USA.

"lll Visitor from Lewis University, Romeoville, Illinois, USA.
}

PACS numbers: 12.15.Ji, 13.85.Qk, 14.70.Hp, 14.80.Bn

misidentified as a lepton, since few other processes in the standard model $(\mathrm{SM})$ produce four isolated leptons. We also unfold our measurement to determine the $\sigma(p \bar{p} \rightarrow Z Z)$ cross section.

After measuring the $t$-channel $Z / \gamma^{*} Z / \gamma^{*}$ cross section, we reinterpret the analysis as a search for the Higgs boson in the four lepton final state, predicted in the SM as a result of electroweak symmetry breaking. Both the ATLAS and CMS experiments at the CERN LHC $p p$ collider have observed a four lepton resonance at a mass of $\sim 125 \mathrm{GeV}$ $[1,2]$ which, when combined with other decay channels, is consistent with the SM Higgs boson.

$Z$ boson pair production was studied at the CERN LEP2 collider by the ALEPH [3], DELPHI [4], L3 [5], and OPAL [6] collaborations in multiple final states, including $e^{+} e^{-} \rightarrow \ell^{+} \ell^{-} \ell^{\prime+} \ell^{\prime-}$, where $\ell$ represents an electron or a muon. The LEP experiments also set limits on anomalous $Z Z Z$ and $Z Z \gamma$ couplings [7].

The Fermilab Tevatron experiments have also searched for and measured the pair production of $Z$ bosons. The D0 Collaboration's analysis of $Z Z \rightarrow \ell^{+} \ell^{-} \ell^{\prime+} \ell^{\prime-}$ production with $1.1 \mathrm{fb}^{-1}$ of $p \bar{p}$ data yielded an upper limit of $4.4 \mathrm{pb}$ on the $Z Z$ production cross section at $95 \%$ C.L. Additionally, limits on anomalous $Z Z Z$ and $Z Z \gamma$ couplings were determined [8]. The D0 Collaboration was the first to observe $Z Z$ production in $p \bar{p}$ collisions in the $\ell^{+} \ell^{-} \ell^{\prime+} \ell^{\prime-}$ final state with $2.7 \mathrm{fb}^{-1}$ of data [9]. The D0 Collaboration has also 
measured the $Z Z$ cross section in the $\ell^{+} \ell^{-} \nu \bar{\nu}$ final state, first with $2.2 \mathrm{fb}^{-1}[10]$ and later with $8.6 \mathrm{fb}^{-1}$ of integrated luminosity, yielding a final measurement of $1.64 \pm$ 0.44 (stat) ${ }_{-0.15}^{+0.13}$ (syst) pb [11]. The CDF Collaboration has analyzed data from $1.9 \mathrm{fb}^{-1}$ of integrated luminosity to study $Z Z$ production, measuring, when combining $\ell^{+} \ell^{-} \ell^{1+} \ell^{\prime-}$ and $\ell^{+} \ell^{-} \nu \bar{\nu}$ channels, a cross section of $\sigma(Z Z)=1.4_{-0.6}^{+0.7}($ stat + syst $) \mathrm{pb} \quad$ [12]. The ATLAS Collaboration has observed $p p \rightarrow Z Z$ production in the four charged lepton final state in $1.0 \mathrm{fb}^{-1}$ of data at $\sqrt{s}=7 \mathrm{TeV}$ [13]. The CMS Collaboration has measured $\sigma(p p \rightarrow Z Z)$ in $5.0 \mathrm{fb}^{-1}$ of data at $\sqrt{s}=7 \mathrm{TeV}$ [14], and has observed the rare decay $Z \rightarrow \ell^{+} \ell^{-} \ell^{\prime+} \ell^{\prime-}$ with a branching fraction in agreement with the SM prediction [15].

This article is an update of the D0 Collaboration's prior $Z Z$ to four charged lepton analysis that measured a cross section of $\sigma(p \bar{p} \rightarrow Z Z)=1.26_{-0.37}^{+0.47}$ (stat) \pm 0.11 (syst) \pm 0.08 (lumi) pb using $6.4 \mathrm{fb}^{-1}$ of integrated luminosity [16]. The result presented here uses 9.6 to $9.8 \mathrm{fb}^{-1}$ of integrated luminosity, and expands electron acceptance in the eeee final state.

\section{DETECTOR}

The D0 detector is described in detail elsewhere [17-20]. The main components are the central tracking system, the calorimeter system, and the muon detectors. The central tracking system is located within a $2 \mathrm{~T}$ solenoidal field and consists of two different trackers. Located closest to the interaction point is the silicon microstrip tracker and surrounding that is the central fiber tracker. The silicon microstrip tracker is an assembly of barrel silicon detectors in the central region, along with largediameter disks in the forward regions for tracking at high pseudorapidity $(\eta)$ [21]. The central fiber tracker consists of eight concentric coaxial barrels each carrying two doublet layers of scintillating fibers. The liquid-argon calorimeter system is housed in three cryostats. The central calorimeter (CC) covers up to $|\eta|=1$, and two end calorimeters (EC) are located in the forward regions, extending coverage to $|\eta|=4$. In the intercryostat region (ICR) between the $\mathrm{CC}$ and EC cryostats, there is a scintillating intercryostat detector between $1.1<|\eta|<1.4$ that recovers some energy from particles passing through the ICR. Closest to the collisions are the electromagnetic (EM) regions of the calorimeter followed by hadronic layers of fine and coarse segmentation.

A muon detection system [22] is located beyond the calorimeters and consists of a layer of tracking detectors and scintillation trigger counters before $1.8 \mathrm{~T}$ toroid magnets, followed by two similar layers after the toroids.

There is a three-level trigger system consisting of a collection of specialized hardware elements, microprocessors, and decision-making algorithms to selectively record the events of most interest.

\section{MONTE CARLO}

We use the PYTHIA [23] Monte Carlo (MC) program to determine the $Z / \gamma^{*} Z / \gamma^{*} \rightarrow \ell^{+} \ell^{-} \ell^{\prime+} \ell^{\prime-}$ signal acceptance and to simulate the migration background. The signal is defined to consist of $Z / \gamma^{*} Z / \gamma^{*}$ pairs where each $Z / \gamma^{*}$ boson has a mass greater than $30 \mathrm{GeV}$. The migration background consists of $Z / \gamma^{*} Z / \gamma^{*}$ events where at least one of the two $Z / \gamma^{*}$ bosons has an invariant mass of less than $30 \mathrm{GeV}$; it enters the signal sample either due to mismeasurement or by misassigning the lepton pairs in the eeee and $\mu \mu \mu \mu$ channels. We include $Z / \gamma^{*} Z / \gamma^{*} \rightarrow$ $\ell^{+} \ell^{-} \tau^{+} \tau^{-}$events where the taus decay into electrons or muons as appropriate to match the final four lepton signature in the signal acceptance. Contributions from $Z Z \rightarrow$ $\tau^{+} \tau^{-} \tau^{+} \tau^{-}$with subsequent decays into muons and electrons are also examined, but found to be negligible. The $Z Z$ transverse momentum $\left(p_{T}\right)$ spectrum is also estimated using SHERPA MC [24], and the difference between the $p_{T}$ spectra from PYTHIA and SHERPA is used as a systematic. The dominant tree-level diagrams for $p \bar{p} \rightarrow$ $Z / \gamma^{*} Z / \gamma^{*} \rightarrow \ell^{+} \ell^{-} \ell^{\prime+} \ell^{\prime-}$ are shown in Fig. 1. The singly resonant $Z$ boson diagram contributes at low mass, and we expect a negligible contribution to the signal yields from this diagram in our analysis.

To estimate the migration background, we generate $Z / \gamma^{*}$ pairs where at least one of the bosons has a mass between 5 and $30 \mathrm{GeV}$, and estimate the cross section of these events using next-to-leading-order (NLO) MC from MCFM [25] with the CTEQ61M PDF set [26].

The $t \bar{t}$ background is estimated using ALPGEN [27] with a top quark mass of $172 \mathrm{GeV}$ and is normalized to an approximate next-to-NLO (NNLO) cross section calculation [28].

Other backgrounds where photons or jets must be misidentified for the event to enter our sample, such as events containing a $Z$ plus jets, are estimated from data as described in Sec. VI.

For the Higgs boson search, we generate SM Higgs boson events with masses between 115 and $200 \mathrm{GeV}$ in $5 \mathrm{GeV}$ increments. We simulate the gluon fusion $(\mathrm{gg} \rightarrow H)$ and $Z H$ associated production $(q \bar{q} \rightarrow Z H)$ processes using PYTHIA. The expected $g g \rightarrow H$ cross section is corrected to
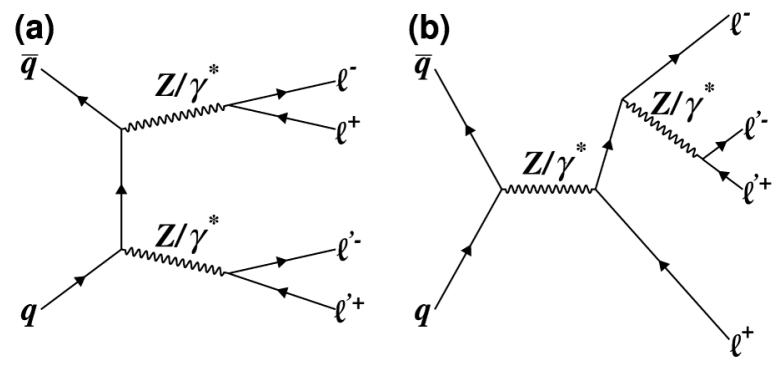

FIG. 1. Feynman diagrams for (a) the $t$-channel tree-level process $q \bar{q} \rightarrow Z Z \rightarrow \ell^{+} \ell^{-} \ell^{\prime+} \ell^{\prime-}$ and (b) the singly resonant process. 
NNLO with next-to-next-to-leading-log resummation of soft gluons [29]. The associated $\mathrm{ZH}$ production cross section is corrected to NNLO [30]. The expected branching fractions for the Higgs boson decay are determined using HDECAY [31].

All of the MC samples are passed through a GEANT [32] simulation of the D0 detector. To account for detector noise and additional $p \bar{p}$ interactions, data from random beam crossings are overlaid onto all MC events to match the instantaneous luminosity distribution of the selected data. The same algorithms used to reconstruct real data events are run on these simulated events.

\section{OBJECT IDENTIFICATION}

All muon candidates are reconstructed either as a muon track reconstructed from hits in both the wire chambers and scintillators in at least one layer of the muon system, or as a narrow energy deposit in the calorimeter system consistent with that expected from a muon passing through the calorimeter that is not associated with tracks in the muon system. Each muon candidate must be matched to a track in the central tracker with a $p_{T}>15 \mathrm{GeV}$, and the track $p_{T}$ is taken as the $p_{T}$ of the muon, $p_{T}^{\mu}$. This track must have an impact parameter consistent with the muon coming from the interaction point. We consider two muon isolation variables: $E_{T}^{\text {trkcone }}$, the scalar sum of the track $p_{T}$ within a cone of $\Delta R \leq 0.5$ [33] about the muon track; and $E_{T}^{\text {halo }}$, the sum of the calorimeter energy in an annulus $0.1<\Delta R \leq$ 0.4 centered on the muon track. If the muon is reconstructed in the muon system, then we impose the requirement that $E_{T}^{\text {trkcone }} / p_{T}^{\mu}<0.25$ and $E_{T}^{\text {halo }} / p_{T}^{\mu}<0.4$. Otherwise, each variable divided by $p_{T}^{\mu}$ must be less than 0.1 .

Different selection requirements apply for electrons identified in the CC $\left(\left|\eta_{d}\right|<1.1\right)$, EC $\left(1.5<\left|\eta_{d}\right|<3.2\right)$, and ICR $\left(1.1<\left|\eta_{d}\right|<1.5\right)$, where $\eta_{d}$ is the pseudorapidity calculated with respect to the center of the detector. In the $\mathrm{CC}$ and EC, electrons must have at least $90 \%$ of their energy found in the EM calorimeter, have $p_{T}>15 \mathrm{GeV}$, and pass a calorimeter isolation requirement. The $p_{T}$ estimate for the CC and EC electrons is based on the energy deposited in the calorimeter. For electrons in the CC, the sum of transverse momenta of the charged central tracks in an annulus of $0.05<\Delta R \leq 0.4$ about the electron, I4, must be less than $4.0 \mathrm{GeV}$. There must either be a track in the central tracker associated with the calorimeter cluster, or hits in the central tracker consistent with a track along the extrapolation of the calorimeter cluster to the interaction point. Finally, the electron must pass a neural net discriminant trained to separate electrons from jets in the $\mathrm{CC}$ using seven shower shape and isolation variables as input.

In the $\mathrm{EC}$ only, we require that the track isolation $\mathrm{I} 4$ be less than $\left(7.0-2.5 \times\left|\eta_{d}\right|\right) \mathrm{GeV}$ or $0.01 \mathrm{GeV}$, whichever is larger. The electron must pass a neural net discriminant trained to separate electrons from jets in the EC using three shower shape and isolation variables as input and an additional chi-square-based shower shape requirement designed to distinguish electrons from jets.

Within the ICR, there is incomplete EM calorimeter coverage, so the electron must pass a minimum EM plus intercryostat detector energy fraction requirement that varies with $\left|\eta_{d}\right|$. The candidate must be matched to a central track with $p_{T}>15 \mathrm{GeV}$ and have a $p_{T}>$ $10 \mathrm{GeV}$ measured in the calorimeter. Additionally, the ICR electron must satisfy two multivariate discriminants designed to reject jet background. Due to the limited energy resolution in the ICR, we use the $p_{T}$ of the track associated with the ICR electron to estimate the ICR electron energy.

Jets are used in the estimation of the instrumental background, as discussed in Sec. VI. In this analysis, we use jets reconstructed from energy deposits in the $\mathrm{CC}, \mathrm{EC}$, and intercryostat detectors using the Run II midpoint cone algorithm [34] with a cone size of $\Delta R=0.5$. The jets must have $p_{T}>15 \mathrm{GeV}$ and $\left|\eta_{d}\right|<3.2$. We apply the standard jet energy scale (JES) corrections [35] to jets in both data and MC.

The missing transverse energy, $\mathbb{E}_{T}$, is calculated using a vector sum of the transverse components of calorimeter energy depositions, with appropriate JES corrections [35]. In the $е e \mu \mu$ and $\mu \mu \mu \mu$ final states, the $\mathbb{E}_{T}$ is corrected for identified muons.

\section{EVENT SELECTION}

To maximize the acceptance, we consider all events that pass the event selection requirements listed below without requiring a specific trigger. The majority of our acceptance comes from events collected by single lepton and dilepton triggers. With four high- $p_{T}$ leptons in this final state, we estimate that the trigger efficiency for the signal is greater than $99.5 \%$ in all channels. This estimate is based on measurements of the single lepton trigger efficiencies in dilepton data with the same instantaneous luminosity and trigger profile as used to make this measurement as well as studies of the increase in efficiency in data from dilepton triggers over the single lepton triggers alone.

\section{A. eeee final state}

All electron candidates have to satisfy the requirements in Sec. IV. We require at least four electron candidates. If there are four CC/EC electron candidates, no ICR electron candidates are considered, and if there are more than four $\mathrm{CC} / \mathrm{EC}$ electron candidates, the highest- $p_{T}$ candidates are used. At least two of the electrons must be in the $\mathrm{CC}$, and if an event has more than one ICR electron, only the leading ICR electron is considered as a lepton candidate. All possible pairings of the selected electrons are considered with no charge requirement imposed, and we require that one of the pairings has di-electron mass $M_{e e}>30 \mathrm{GeV}$ for both di-electrons. Additionally, there must be $\Delta R>0.5$ between any ICR electron and any CC and EC electrons, or 
the ICR electron is not considered. Because the instrumental background contamination is expected to vary significantly depending on the number of central electrons, the eeee channel is then divided into four subchannels that depend on the number of electrons in the CC, $N_{\mathrm{CC}}$, the EC, $N_{\mathrm{EC}}$, and in the ICR, $N_{\text {ICR }}: N_{\mathrm{CC}}=4, \quad N_{\mathrm{CC}}=2$ with $N_{\mathrm{EC}}=2, N_{\mathrm{CC}}=3$ with $N_{\mathrm{EC}}=1$, and $N_{\mathrm{CC}} \geq 2$ with $N_{\text {ICR }}=1$. Since we do not use the muon system in eeee event reconstruction, we include events where the muon system was not fully operational. This leads to a slightly higher integrated luminosity in the eeee final state compared to the $e e \mu \mu$ and $\mu \mu \mu \mu$ final states.

\section{B. $e$ e $\mu \mu$ channel}

The $e e \mu \mu$ channel is divided into three subchannels that depend on the number of electrons in the CC: $N_{\mathrm{CC}}=2$, $N_{\mathrm{CC}}=1$, and $N_{\mathrm{CC}}=0$. No ICR electrons are used in this channel. As in the eeee final state, we apply this splitting because the instrumental background contamination varies significantly depending on the number of central electrons. We require at least two electrons and two muons; if there are more leptons in the event, only the highest- $p_{T}$ leptons of each type are used. To reject cosmic ray background, the cosine of the angle between the muons must satisfy $\cos \alpha<0.96$, and the acoplanarity [36] between the two muons must be greater than 0.05 radians. We further require $\left|\Delta z_{\mathrm{DCA}}\right|<3.0 \mathrm{~cm}$ between the muon tracks, where $z_{\mathrm{DCA}}$ refers to the location along the beam axis where the track has its distance of closest approach to the beam line. Also, we impose the requirement that $\Delta R>0.2$ between all possible electron-muon pairings. Both the muon pair and electron pair invariant masses must exceed $30 \mathrm{GeV}$. There is no opposite charge requirement placed on the lepton pairs in order to maximize acceptance.

\section{C. $\mu \boldsymbol{\mu} \mu \boldsymbol{\mu}$ final state}

In the four muon final state, there must be at least four muon candidates satisfying the requirements in Sec. IV, and at least two of the muons must be matched to tracks found in the muon system. The four muon system must be charge neutral $\left(\sum_{i=1}^{4} q_{i}=0\right)$, and only oppositely charged pairs are considered as $Z$ boson candidates. If more than four muons are reconstructed in the event, we consider only the four highest- $p_{T}$ muons. We further require $\left|\Delta z_{\mathrm{DCA}}\right|<$ $3.0 \mathrm{~cm}$ between all muons and that one of the two possible combinations for defining the dimuon pairs gives an invariant mass $M_{\mu \mu}>30 \mathrm{GeV}$ for both dimuon pairs.

\section{INSTRUMENTAL BACKGROUND}

The instrumental background primarily arises from $Z(\rightarrow \ell \ell)+$ jets and $Z(\rightarrow \ell \ell)+\gamma+$ jets production (with smaller contributions from $W Z+$ jets, $W W+$ jets, $W+$ jets, and multijet production with $\geq 4$ jets). These events contaminate the four-lepton channels when a jet is falsely reconstructed as an isolated lepton, either due to mismeasurement of the jet properties or because a real lepton was produced from the decay of a hadron in the jet. $Z(\rightarrow \ell \ell)+\gamma+$ jets production where a photon and a jet are misidentified as an electron contaminates the eeee and $е e \mu \mu$ channels.

We estimate the instrumental background using the data. We first find the probability for a jet to be misidentified as a lepton, $P_{j \ell}$. A tag and probe method is used to determine $P_{j \ell}$ where di-jet activity is considered with jet $p_{T}>$ $15 \mathrm{GeV}$. The tagged jet must be associated with a jet that fired a single jet trigger and be the highest- $p_{T}$ jet in the event. We then look for a probe jet with $|\Delta \phi|>3.0$ with respect to the tag jet, where $\phi$ is the azimuthal angle. To suppress contamination from $W+$ jet events, we require $\mathscr{E}_{T}<20 \mathrm{GeV}$ in the tag and probe sample. The probe jets form the denominator of the $P_{j \ell}$ calculation.

To calculate the numerator of the $P_{j e}$ estimate, we first find all good electrons in the event with a $p_{T}>15 \mathrm{GeV}$. We then select those electrons that satisfy the same criteria imposed on the probe jets, noted above. The $P_{j e}$ estimate is parametrized as a function of the jet $p_{T}$ and $\eta_{d}$.

The $P_{j \mu}$ estimate is determined using a similar method. The tagged jet is defined as was done for electron events. In the numerator, rather than an electron, we use any muon that has $|\Delta \phi|>3.0$ from the tag jet. We then take $P_{j \mu}$ as the number of muons divided by the number of probe jets in the sample. The $P_{j \mu}$ estimate is parametrized in terms of $p_{T}$ and $\eta$.

The $P_{j \ell}$ estimates for both electrons and muons are on the order of $10^{-3}$.

To estimate the instrumental background for the eeee final state, $P_{j e}$ is applied to events with three reconstructed electrons and one or more jets. The jet kinematics are used to model the electron kinematics in the event. This method accounts for events where either a photon or a jet is misreconstructed as one electron and a jet is misreconstructed as the other. This method overestimates the background from events with two real electrons and two jets misreconstructed as electrons. To determine the rate, we look at events with two reconstructed electrons and two or more reconstructed jets and apply $P_{j e}$ to both jets. The number of $e e$ plus two jet events after $P_{j e}$ is applied to both jets is found to be negligible, so only eee + jet events are used to model the instrumental background distributions in the eeee final state.

The instrumental background in the $e e \mu \mu$ channel is calculated from two different contributions. The first contribution is from events with $e \mu \mu$ plus one or more jets, where we apply $P_{j e}$ to the jet. This method gives an estimate of a background due to $Z(\rightarrow \mu \mu)+$ jets and $Z(\rightarrow \mu \mu)+\gamma+$ jets where a jet has been reconstructed as an electron. We also consider the $e e$ plus two jets or more case, where we apply $P_{j \mu}$ to the jets. This method 
TABLE I. Contributions from non-negligible backgrounds in the eeee subchannels, plus expected $t$-channel ZZ and Higgs boson signals and number of observed events. Uncertainties are statistical followed by systematic.

\begin{tabular}{|c|c|c|c|c|}
\hline & $\begin{array}{l}2 \mathrm{CC} \\
2 \mathrm{EC}\end{array}$ & $\begin{array}{l}3 \mathrm{CC} \\
1 \mathrm{EC}\end{array}$ & $4 \mathrm{CC}$ & $\begin{array}{c}\geq 2 \mathrm{CC} \\
1 \mathrm{ICR}\end{array}$ \\
\hline Instrumental backg. & $0.15 \pm 0.01 \pm 0.03$ & $0.12 \pm 0.01 \pm 0.02$ & $0.05 \pm 0.01 \pm 0.01$ & $0.29 \pm 0.04_{-012}^{+0.03}$ \\
\hline Migration & $0.014 \pm 0.001 \pm 0.002$ & $0.023 \pm 0.001 \pm 0.004$ & $0.025 \pm 0.001 \pm 0.004$ & $0.024 \pm 0.001 \pm 0.003$ \\
\hline Total non- $Z Z$ background & $0.17 \pm 0.01 \pm 0.03$ & $0.14 \pm 0.01 \pm 0.02$ & $0.08 \pm 0.01 \pm 0.01$ & $0.32 \pm 0.04_{-0.12}^{+0.03}$ \\
\hline Expected $t$-channel $Z / \gamma^{*} Z / \gamma^{*}$ & $0.48 \pm 0.01 \pm 0.07$ & $1.14 \pm 0.01 \pm 0.17$ & $1.03 \pm 0.01 \pm 0.15$ & $1.47 \pm 0.01 \pm 0.19$ \\
\hline Expected $g g \rightarrow H M_{H}=125 \mathrm{GeV}$ & $<0.001$ & 0.001 & 0.004 & 0.002 \\
\hline Expected $Z H M_{H}=125 \mathrm{GeV}$ & 0.003 & 0.006 & 0.010 & 0.008 \\
\hline Total Higgs boson $M_{H}=125 \mathrm{GeV}$ & 0.003 & 0.007 & 0.014 & 0.010 \\
\hline Observed events & 0 & 1 & 2 & 2 \\
\hline
\end{tabular}

TABLE II. Contributions from non-negligible backgrounds in the $e e \mu \mu$ subchannels, plus expected signal and number of observed events. Uncertainties are statistical followed by systematic.

\begin{tabular}{lccc}
\hline \hline & $0 \mathrm{CC}$ & $1 \mathrm{CC}$ & $2 \mathrm{CC}$ \\
\hline Instrumental backg. & $0.11 \pm 0.01 \pm 0.03$ & $0.21 \pm 0.01 \pm 0.04$ & $0.27 \pm 0.01 \pm 0.04$ \\
$t \bar{t}$ & $\left(0.2_{-0.1}^{+0.3} \pm 0.6\right) \times 10^{-2}$ & $\left(1.0_{-0.3}^{+0.5} \pm 0.2\right) \times 10^{-2}$ & $\left(0.3_{-0.1}^{+0.2} \pm 0.3\right) \times 10^{-2}$ \\
Migration & $\left(2.1_{-0.7-1.0}^{+0.9+0.3}\right) \times 10^{-3}$ & $\left(5.0 \pm 0.8_{-1.4}^{+0.6}\right) \times 10^{-3}$ & $\left(4.8_{-0.5}^{+0.6} \pm 1.0\right) \times 10^{-3}$ \\
Cosmic rays & $<0.001$ & $<0.003$ & $<0.006$ \\
Total non- $Z Z$ background & $0.12 \pm 0.01 \pm 0.03$ & $0.23 \pm 0.01 \pm 0.04$ & $0.27 \pm 0.01 \pm 0.04$ \\
Expected $t$-channel $Z / \gamma^{*} Z / \gamma^{*}$ & $0.43 \pm 0.01 \pm 0.06$ & $2.37 \pm 0.02 \pm 0.28$ & $4.13 \pm 0.03 \pm 0.49$ \\
Expected $g g \rightarrow H M_{H}=125 \mathrm{GeV}$ & $<0.001$ & 0.002 & 0.007 \\
Expected $Z H M_{H}=125 \mathrm{GeV}$ & 0.001 & 0.015 & 0.036 \\
Total Higgs boson $M_{H}=125 \mathrm{GeV}$ & 0.002 & 0.017 & 0.043 \\
Observed events & 2 & 1 & 2 \\
\hline \hline
\end{tabular}

gives an estimate of the background due to $Z(\rightarrow e e)+$ jets where the jets can contain muons.

The $P_{j \mu}$ is applied to jets in $\mu \mu$ plus two or more jets data to determine the instrumental background for the $\mu \mu \mu \mu$ channel.

Background estimates derived from the above method can be found in Tables I, II, and III in each final state.

TABLE III. Contributions from non-negligible backgrounds in the $\mu \mu \mu \mu$ channel, plus expected $t$-channel ZZ and Higgs boson signal and number of observed events. Uncertainties are statistical followed by systematic.

\begin{tabular}{lc}
\hline \hline & Number of events \\
\hline Instrumental backg. & $0.12 \pm 0.01_{-0.05}^{+0.07}$ \\
Migration & $\left(0.34 \pm 0.02_{-0.04}^{+0.07}\right) \times 10^{-1}$ \\
Cosmic rays & $<0.01$ \\
Total non- $Z Z$ background & $0.15 \pm 0.01_{-0.05}^{+0.07}$ \\
Expected $t$-channel $Z / \gamma^{*} Z / \gamma^{*}$ & $4.26 \pm 0.02 \pm 0.43$ \\
Expected $g g \rightarrow H M_{H}=125 \mathrm{GeV}$ & 0.007 \\
Expected $Z H M_{H}=125 \mathrm{GeV}$ & 0.033 \\
Total Higgs boson $M_{H}=125 \mathrm{GeV}$ & 0.040 \\
Observed events & 3 \\
\hline \hline
\end{tabular}

\section{SYSTEMATIC UNCERTAINTIES}

The following factors contribute to the systematic uncertainty on this measurement. We apply a $1 \%$ trigger efficiency uncertainty. Lepton identification uncertainties are calculated by studying $Z \rightarrow \ell \ell$ events; lepton identification uncertainties of $3.7 \%$ per $\mathrm{CC}$ and EC electron, $6 \%$ per ICR electron, and $3.2 \%$ per muon are used. There is a $10 \%-50 \%$ systematic uncertainty on the instrumental background expectation in the various final states that is due to observed variations in $P_{j \ell}$ when changing selection requirements for the di-jet sample as well as limited statistics in the data samples used. We assign $20 \%$ uncertainty to the $t \bar{t}$ background. This covers uncertainty on the theoretical production rate of $7 \%$ for $m_{\text {top }}=172 \mathrm{GeV}$ [28], plus variation in the cross section due to uncertainty on the top quark mass, and also that on the rate at which the $b$ quark from top quark decays is misidentified as an isolated lepton. We estimate a PDF uncertainty of $2.5 \%$ on all MC samples. We assign a $7.1 \%$ uncertainty on the $Z Z$ cross section used to estimate the migration background and the ZZ background to the Higgs boson search. A systematic uncertainty of $6.1 \%$ is assessed on the luminosity measurement [37]. We assess a systematic uncertainty on the $Z Z p_{T}$ distribution by reweighting the PYTHIA $Z Z p_{T}$ to match a 
TABLE IV. Acceptance $\times$ efficiency for the eeee subchannels, for $Z Z \rightarrow$ eeee and $Z Z \rightarrow e e \tau \tau$ decays. Uncertainties are statistical followed by systematic.

\begin{tabular}{|c|c|c|}
\hline Channel & eeee & $e e \tau \tau$ \\
\hline $2 \mathrm{CC}, 2 \mathrm{EC}$ & $0.025 \pm 0.001 \pm 0.004$ & $0.0002 \pm 0.0001 \pm 0.0001$ \\
\hline $3 \mathrm{CC}, 1 \mathrm{EC}$ & $0.059 \pm 0.001 \pm 0.011$ & $0.0006 \pm 0.0001 \pm 0.0001$ \\
\hline $4 \mathrm{CC}$ & $0.053 \pm 0.001 \pm 0.009$ & $0.0007 \pm 0.0001 \pm 0.0001$ \\
\hline$\geq 2 \mathrm{CC}, 1 \mathrm{ICR}$ & $0.076 \pm 0.001 \pm 0.012$ & $0.0007 \pm 0.0001 \pm 0.0001$ \\
\hline
\end{tabular}

TABLE V. Acceptance $\times$ efficiency for the $e e \mu \mu$ subchannels, for $Z Z \rightarrow e e \mu \mu$, $Z Z \rightarrow e e \tau \tau$, and $Z Z \rightarrow \mu \mu \tau \tau$ decays. Uncertainties are statistical followed by systematic.

\begin{tabular}{lccc}
\hline \hline Channel & $e e \mu \mu$ & $e e \tau \tau$ & $\mu \mu \tau \tau$ \\
\hline $0 \mathrm{CC}$ & $0.011 \pm 0.001 \pm 0.001$ & $0.0001 \pm 0.0001 \pm 0.0001$ & $0.0002 \pm 0.0001 \pm 0.0001$ \\
$1 \mathrm{CC}$ & $0.063 \pm 0.001 \pm 0.007$ & $0.0007 \pm 0.0001 \pm 0.0001$ & $0.0007 \pm 0.0001 \pm 0.0001$ \\
$2 \mathrm{CC}$ & $0.110 \pm 0.001 \pm 0.012$ & $0.0014 \pm 0.0001 \pm 0.0002$ & $0.0019 \pm 0.0001 \pm 0.0002$ \\
\hline \hline
\end{tabular}

distribution derived from SHERPA MC [24]. The $Z Z p_{T}$ systematic is between $1 \%$ and $7 \%$ for signal $t$-channel $Z Z$ events, but has up to a $40 \%$ effect on the migration background. We also assess systematic uncertainties on the muon and electron energy resolution [38], which lead to an uncertainty on the cross section measurements and Higgs boson production limits of less than $2 \%$. There is a $2 \%$ uncertainty on the ratio of $\sigma\left(p \bar{p} \rightarrow Z / \gamma^{*} Z / \gamma^{*}\right)$ to $\sigma(p \bar{p} \rightarrow Z Z)$ from scale uncertainties. For the Higgs boson search, we assess a theoretical uncertainty on the expected gluon fusion and $\mathrm{ZH}$ associated cross sections of $10.9 \%$ and $6.2 \%$, respectively $[29,30]$.

\section{CROSS SECTION MEASUREMENT}

The data are used to measure the production cross section $p \bar{p} \rightarrow Z Z$ at $\sqrt{s}=1.96 \mathrm{TeV}$. The integrated luminosities analyzed for the three channels are 9.8, 9.6, and $9.6 \mathrm{fb}^{-1}$ for the eeee, eе $\mu \mu$, and $\mu \mu \mu \mu$ channels, respectively. A summary of the signal and background event expectations are included in Tables I, II, and III for the three channels.

We observe five eeee candidate events, five $e е \mu \mu$ candidate events, and three $\mu \mu \mu \mu$ candidate events, for 13 data events total, with a total of $16.8 \pm 1.9$ (stat + syst + lumi) expected events.

A negative log-likelihood function is constructed by taking as input the expected signal acceptance, the number of expected background events, and the number

TABLE VI. Acceptance $\times$ efficiency for the $\mu \mu \mu \mu$ channel, for $Z Z \rightarrow \mu \mu \mu \mu$ and $Z Z \rightarrow \mu \mu \tau \tau$ decays. Uncertainties are statistical followed by systematic.

\begin{tabular}{lc}
\hline \hline$\mu \mu \mu \mu$ & $\mu \mu \tau \tau$ \\
\hline $0.224 \pm 0.002 \pm 0.022$ & $0.0032 \pm 0.0002 \pm 0.0003$ \\
\hline \hline
\end{tabular}
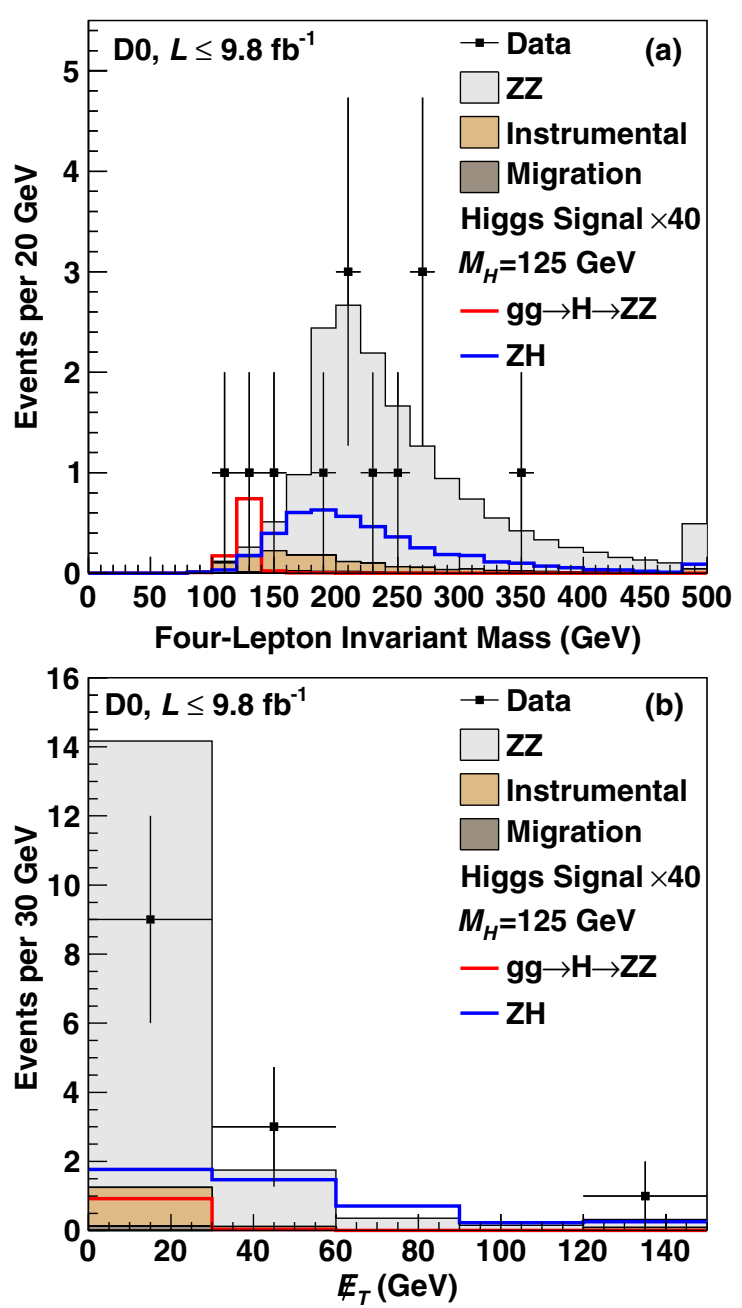

FIG. 2 (color online). Distributions of (a) the four lepton invariant mass and (b) the $\mathbb{E}_{T}$ in data, and of expected signal and background. The Higgs boson signal for $M_{H}$ of $125 \mathrm{GeV}$ is shown scaled by a factor of 40 . 
TABLE VII. Expected numbers of Higgs boson events for each mass point for the given production and decay mode. The $H \rightarrow \gamma \gamma, H \rightarrow \mu \mu$, and $H \rightarrow Z \gamma$ contributions are summed together in the $H \rightarrow$ other decays column.

\begin{tabular}{lcccccc}
\hline \hline & \multirow{2}{*}{$g \rightarrow H$} & \multicolumn{2}{c}{$q \bar{q} \rightarrow Z H$} \\
$M_{H}(\mathrm{GeV})$ & $H \rightarrow Z Z$ & $H \rightarrow W W$ & $H \rightarrow Z Z$ & $H \rightarrow \tau \tau$ & $H \rightarrow$ other & Total \\
\hline 115 & 0.009 & 0.016 & 0.013 & 0.060 & 0.008 & 0.106 \\
120 & 0.013 & 0.026 & 0.017 & 0.052 & 0.006 & 0.113 \\
125 & 0.024 & 0.040 & 0.024 & 0.043 & 0.005 & 0.137 \\
130 & 0.049 & 0.058 & 0.039 & 0.035 & 0.004 & 0.184 \\
135 & 0.090 & 0.066 & 0.047 & 0.025 & 0.003 & 0.232 \\
140 & 0.138 & 0.077 & 0.055 & 0.018 & 0.003 & 0.291 \\
145 & 0.185 & 0.088 & 0.061 & 0.013 & 0.002 & 0.348 \\
150 & 0.210 & 0.092 & 0.059 & 0.008 & 0.001 & 0.371 \\
155 & 0.196 & 0.099 & 0.049 & 0.004 & 0.001 & 0.348 \\
160 & 0.112 & 0.100 & 0.026 & 0.002 & 0.000 & 0.240 \\
165 & 0.059 & 0.097 & 0.012 & 0.001 & 0.000 & 0.169 \\
170 & 0.062 & 0.088 & 0.012 & 0.000 & 0.000 & 0.162 \\
175 & 0.082 & 0.086 & 0.015 & 0.000 & 0.000 & 0.183 \\
180 & 0.148 & 0.078 & 0.027 & 0.000 & 0.000 & 0.254 \\
185 & 0.348 & 0.068 & 0.063 & 0.000 & 0.000 & 0.478 \\
190 & 0.440 & 0.058 & 0.077 & 0.000 & 0.000 & 0.575 \\
195 & 0.467 & 0.051 & 0.082 & 0.000 & 0.000 & 0.600 \\
200 & 0.468 & 0.046 & 0.083 & 0.000 & 0.000 & 0.597 \\
\hline \hline
\end{tabular}

of observed events in each of the subchannels. The signal acceptance times efficiency for each channel is shown in Tables IV, V, and VI. The branching ratio for each channel is calculated using the relevant $Z$ boson branching ratios from Ref. [39]. The cross section, $\sigma$, is varied to minimize the negative log-likelihood, which gives $\sigma(p \bar{p} \rightarrow$ $\left.Z / \gamma^{*} Z / \gamma^{*}\right)=1.26_{-0.36}^{+0.44}(\text { stat })_{-0.15}^{+0.17}$ (syst) \pm 0.08 (lumi) pb for

TABLE VIII. Expected and observed 95\% C.L. upper limits on the SM Higgs boson production cross section relative to the value expected in the SM.

\begin{tabular}{lcc}
\hline \hline$M_{H}(\mathrm{GeV})$ & Expected & Observed \\
\hline 115 & 57.3 & 78.9 \\
120 & 54.9 & 60.6 \\
125 & 42.8 & 42.3 \\
130 & 30.6 & 33.5 \\
135 & 21.5 & 21.0 \\
140 & 16.2 & 18.2 \\
145 & 13.4 & 13.9 \\
150 & 12.4 & 12.1 \\
155 & 13.4 & 14.2 \\
160 & 20.8 & 20.6 \\
165 & 29.6 & 28.3 \\
170 & 32.3 & 39.0 \\
175 & 30.4 & 28.4 \\
180 & 22.9 & 19.6 \\
185 & 13.3 & 9.7 \\
190 & 11.8 & 8.6 \\
195 & 11.8 & 9.5 \\
200 & 12.4 & 9.9 \\
\hline \hline
\end{tabular}

$M\left(Z / \gamma^{*}\right)>30 \mathrm{GeV}$. We then calculate the ratio of $\sigma\left(p \bar{p} \rightarrow Z / \gamma^{*} Z / \gamma^{*}\right)$ to $\sigma(p \bar{p} \rightarrow Z Z)$ for this mass region using MCFM [25], and from this correction determine the $p \bar{p} \rightarrow Z Z$ cross section to be $1.05_{-0.30}^{+0.37}(\text { stat })_{-0.12}^{+0.14} \times$ (syst) \pm 0.06 (lumi) $\mathrm{pb}$. We combine this measurement with the $p \bar{p} \rightarrow Z Z$ cross section measured in the $\ell^{+} \ell^{-} \nu \bar{\nu}$ final state using data from the D0 detector [11], giving a total combined $p \bar{p} \rightarrow Z Z$ cross section of $1.32_{-0.25}^{+0.29}$ (stat) \pm 0.12 (syst) \pm 0.04 (lumi) pb. The measured cross section values are consistent with the SM expectation of $1.43 \pm$ $0.10 \mathrm{pb}[25]$.

\section{HIGGS BOSON PRODUCTION LIMITS}

The main Higgs boson production mechanisms that can result in four final state charged leptons are gluon fusion and $\mathrm{ZH}$ associated production.

For Higgs boson events produced through gluon fusion, final states with four charged leptons arise from the decay $H \rightarrow Z Z$, where both $Z$ bosons then decay leptonically. As all of the decay products of the Higgs boson in this decay are well measured, the best discriminating variable between the gluon fusion Higgs boson signal and the backgrounds is the four lepton invariant mass.

In the case of associated $\mathrm{ZH}$ production, two of the leptons in each event can come from the decay of the associated $Z$ boson, so Higgs decay modes with two or more final state leptons will contribute to our signal. The majority of the $Z H$ signal arises from $H \rightarrow \tau^{+} \tau^{-}$, $H \rightarrow W W$, and $H \rightarrow Z Z$ decays. We expect large $\not E_{T}$ in these events, due to the neutrinos from the $\tau$ and $W$ boson 

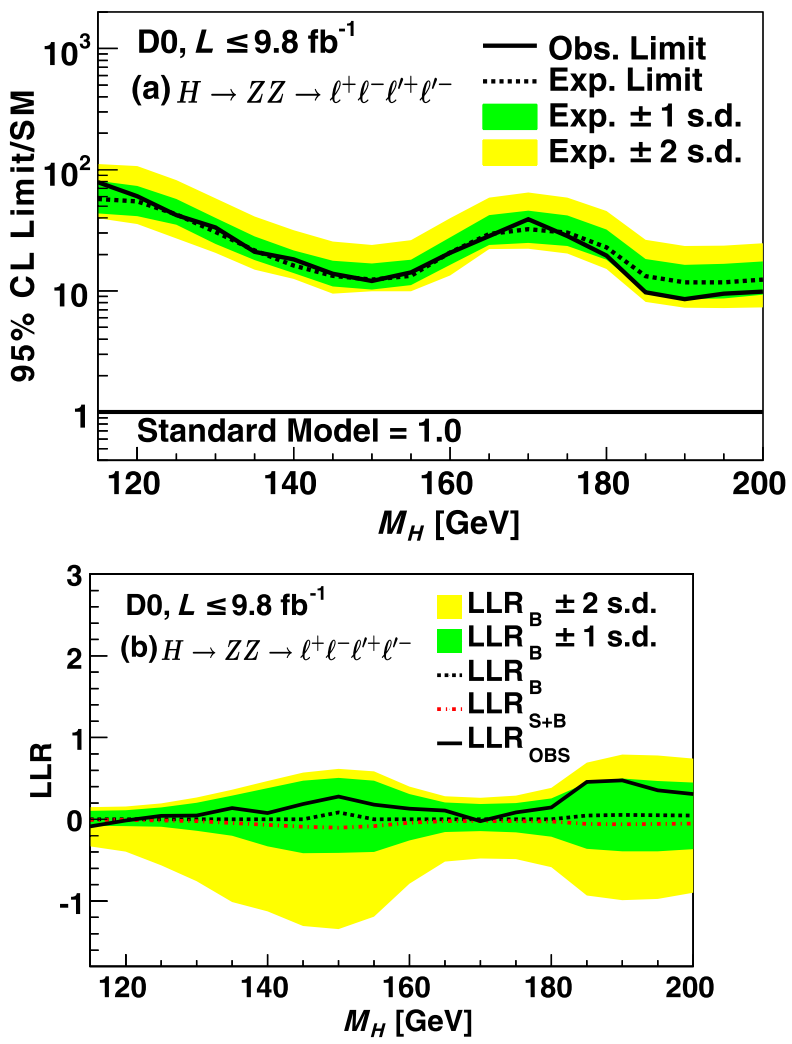

FIG. 3 (color online). The (a) expected and observed 95\% C.L. upper limits on the SM Higgs boson production cross section relative to the value expected in the $\mathrm{SM}$, and the (b) loglikelihood ratio for all four lepton channels combined.

decays, as well as in events where one $Z$ boson from the $H \rightarrow Z Z$ decays to neutrinos.

We therefore set limits on SM Higgs boson production using the four lepton invariant mass and the $\mathbb{E}_{T}$. The four lepton mass and $\mathscr{E}_{T}$ are shown in Fig. 2, with the expected Higgs boson signal distributions for a Higgs boson mass, $M_{H}$, of $125 \mathrm{GeV}$. Additional differential distributions are provided in the Appendix. The expected yields for each production and decay mode for each Higgs boson mass considered are shown in Table VII. For events with $\not ̈_{T}<$ $30 \mathrm{GeV}$, the four lepton mass is used to discriminate the Higgs boson signal from all backgrounds; in events with $\mathbb{E}_{T} \geq 30 \mathrm{GeV}$, the $\mathbb{E}_{T}$ is used. For the Higgs boson search, the $t$-channel $Z / \gamma^{*} Z / \gamma^{*}$ background is fixed to the SM expectation.

We find no evidence of SM Higgs boson production and proceed to set limits. We consider potential $M_{H}$ values between 115 and $200 \mathrm{GeV}$, in $5 \mathrm{GeV}$ increments. We calculate limits on the SM Higgs boson production cross section using a modified frequentist approach [40-42]. A log-likelihood ratio (LLR) test statistic is formed using the Poisson probabilities for estimated background yields, the expected signal acceptance, and the number of observed events for each considered Higgs boson mass hypothesis. The confidence levels are derived by integrating the LLR distribution in pseudoexperiments using both the signal-plus-background hypothesis $\left(C L_{s+b}\right)$ and the background-only hypothesis $\left(C L_{b}\right)$. The excluded production cross section is taken to be the cross section for which the confidence level for signal, $C L_{s}=C L_{s+b} / C L_{b}$, is less than or equal to 0.05 .

The calculated limits are listed in Table VIII. At $M_{H}=125 \mathrm{GeV}$, we expect to set a limit of 42.8 times the SM cross section at the $95 \%$ C.L., and observe a limit of 42.3 times the SM cross section. The limits vs $M_{H}$ are shown in Fig. 3, along with the associated LLR distribution.

\section{CONCLUSIONS}

We have measured the production cross section for $p \bar{p} \rightarrow Z / \gamma^{*} Z / \gamma^{*}$ with $M\left(Z / \gamma^{*}\right)>30 \mathrm{GeV}$ to be $1.26_{-0.36}^{+0.44}(\text { stat })_{-0.15}^{+0.17}$ (syst) \pm 0.08 (lumi) pb. We correct this measurement by the expected ratio of $\sigma(p \bar{p} \rightarrow$ $\left.Z / \gamma^{*} Z / \gamma^{*}\right)$ to $\sigma(p \bar{p} \rightarrow Z Z)$ for this mass region and obtain a $p \bar{p} \rightarrow Z Z$ cross section of $1.05_{-0.30}^{+0.37}(\text { stat })_{-0.12}^{+0.14} \times$ (syst) \pm 0.06 (lumi) pb. We also searched for the Higgs boson in the four lepton final state, assuming that the $t$-channel $Z Z$ pair is produced with the cross section predicted by the SM. At $M_{H}=125 \mathrm{GeV}$, we expect a limit of 42.8 times the SM cross section, and set a limit of 42.3 times the SM cross section at the $95 \%$ C.L.

\section{ACKNOWLEDGMENTS}

We thank the staffs at Fermilab and collaborating institutions, and acknowledge support from the DOE and NSF (USA); CEA and CNRS/IN2P3 (France); MON, NRC KI and RFBR (Russia); CNPq, FAPERJ, FAPESP and FUNDUNESP (Brazil); DAE and DST (India); Colciencias (Colombia); CONACyT (Mexico); NRF (Korea); FOM (The Netherlands); STFC and the Royal Society (United Kingdom); MSMT and GACR (Czech Republic); BMBF and DFG (Germany); SFI (Ireland); The Swedish Research Council (Sweden); and CAS and CNSF (China).

\section{APPENDIX: DIFFERENTIAL DISTRIBUTIONS}

Figures 4-9 show differential distributions of the events used in the $t$-channel $Z Z$ cross section measurement and Higgs boson search. Some of these distributions are kinematic properties of dilepton systems; in the $e e \mu \mu$ final state, the pairings of $e e$ and $\mu \mu$ are always used. In the eeee and $\mu \mu \mu \mu$ final states, there may be multiple combinations passing our selection requirements. If there are multiple passing combinations, we use the combination that yields a dilepton pair with an invariant mass closest to the nominal $Z$ boson mass of $91.2 \mathrm{GeV}$ [39]. Figure 4 shows the dilepton invariant mass and the $p_{T}$ of the four lepton system. The $p_{T}$ and $\eta_{d}$ distributions for each lepton in our events are shown in Figs. 5 and 6, respectively. The $Z / \gamma^{*} p_{T}$ distributions for the highest- $p_{T}$ (leading) and 

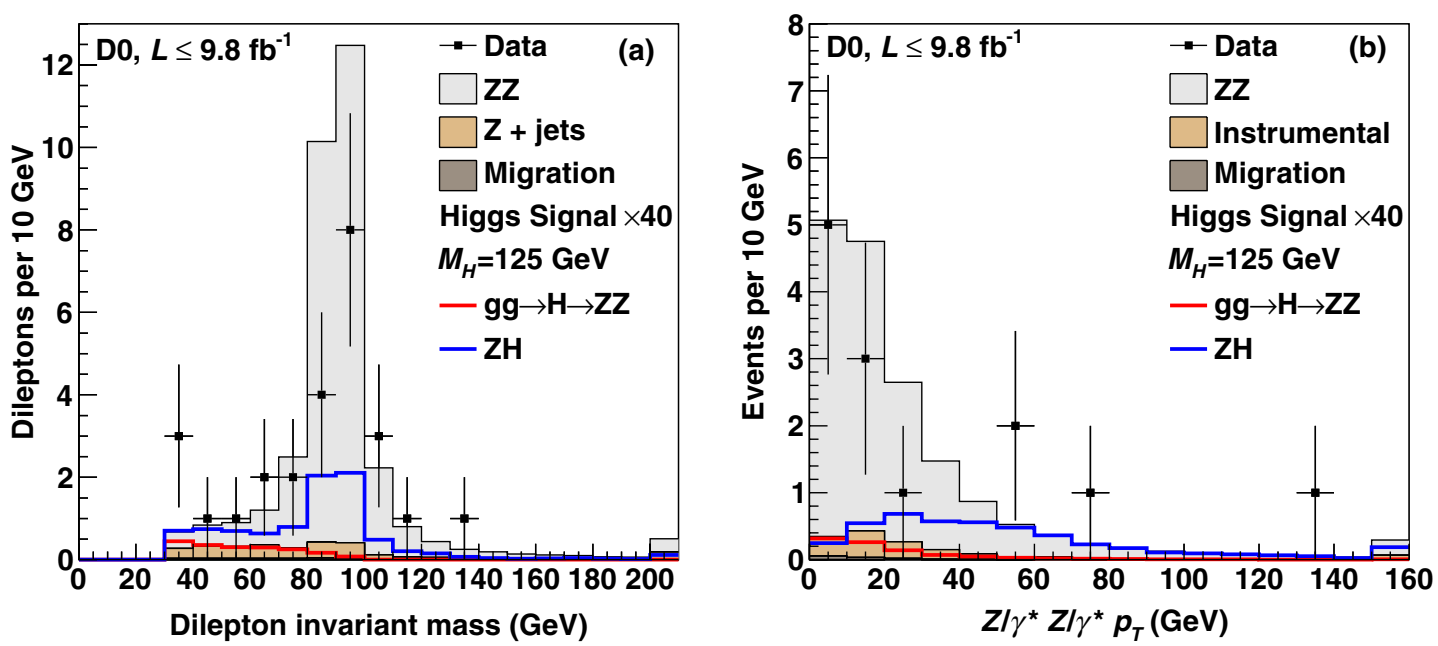

FIG. 4 (color online). Distributions of (a) the dilepton invariant mass and (b) the transverse momentum of the four lepton system in data, expected signal and background. There are two entries per event in the dilepton invariant mass distribution. The Higgs boson signal for $M_{H}$ of $125 \mathrm{GeV}$ is shown scaled by a factor of 40 .
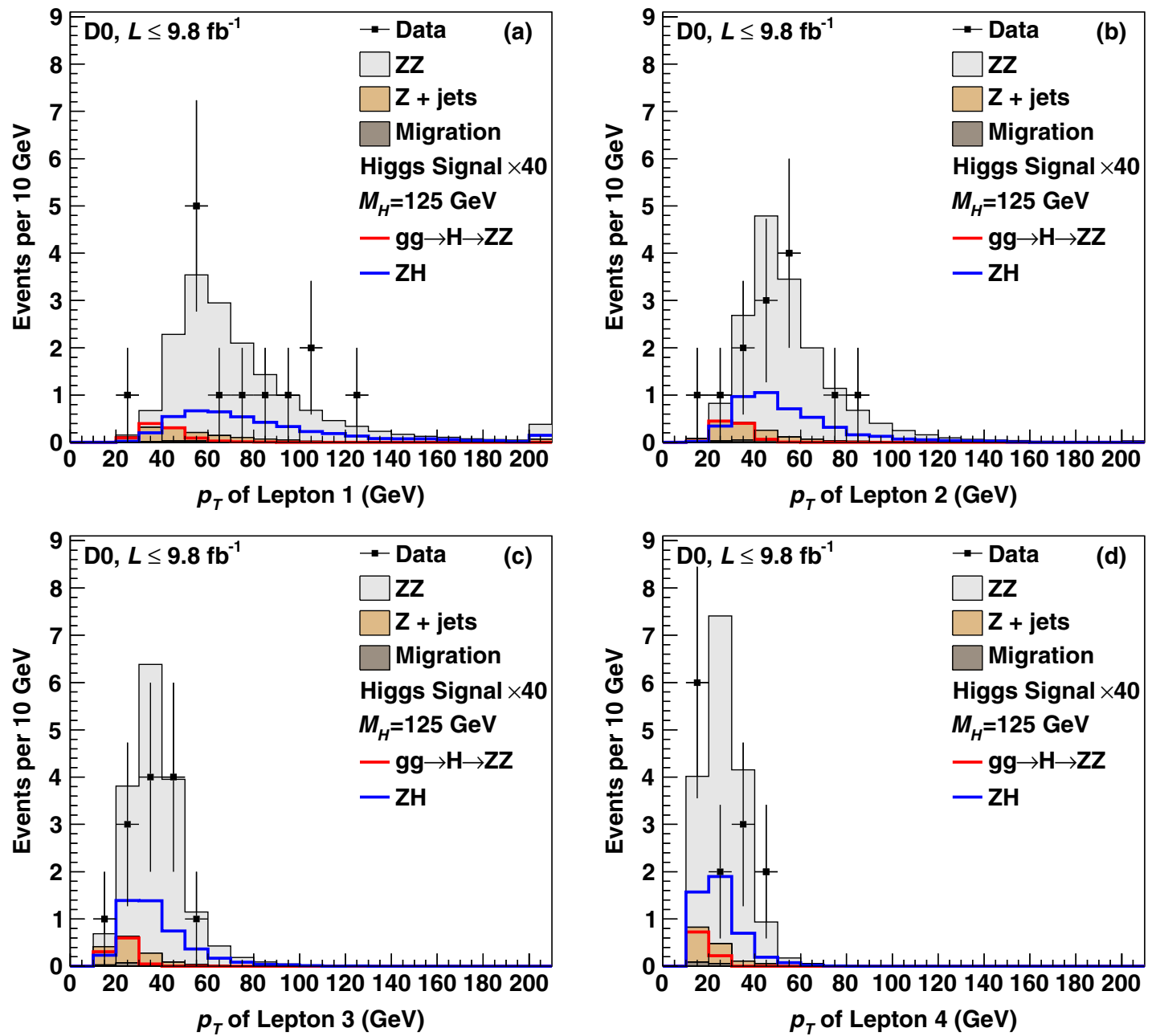

FIG. 5 (color online). Distributions of the transverse momentum in data, expected signal, and backgrounds for the (a) highest- $p_{T}$, (b) second-highest- $p_{T}$, (c) third-highest- $p_{T}$, and (d) lowest- $p_{T}$ leptons in each event. The Higgs boson signal for $M_{H}$ of $125 \mathrm{GeV}$ is shown scaled by a factor of 40 . 

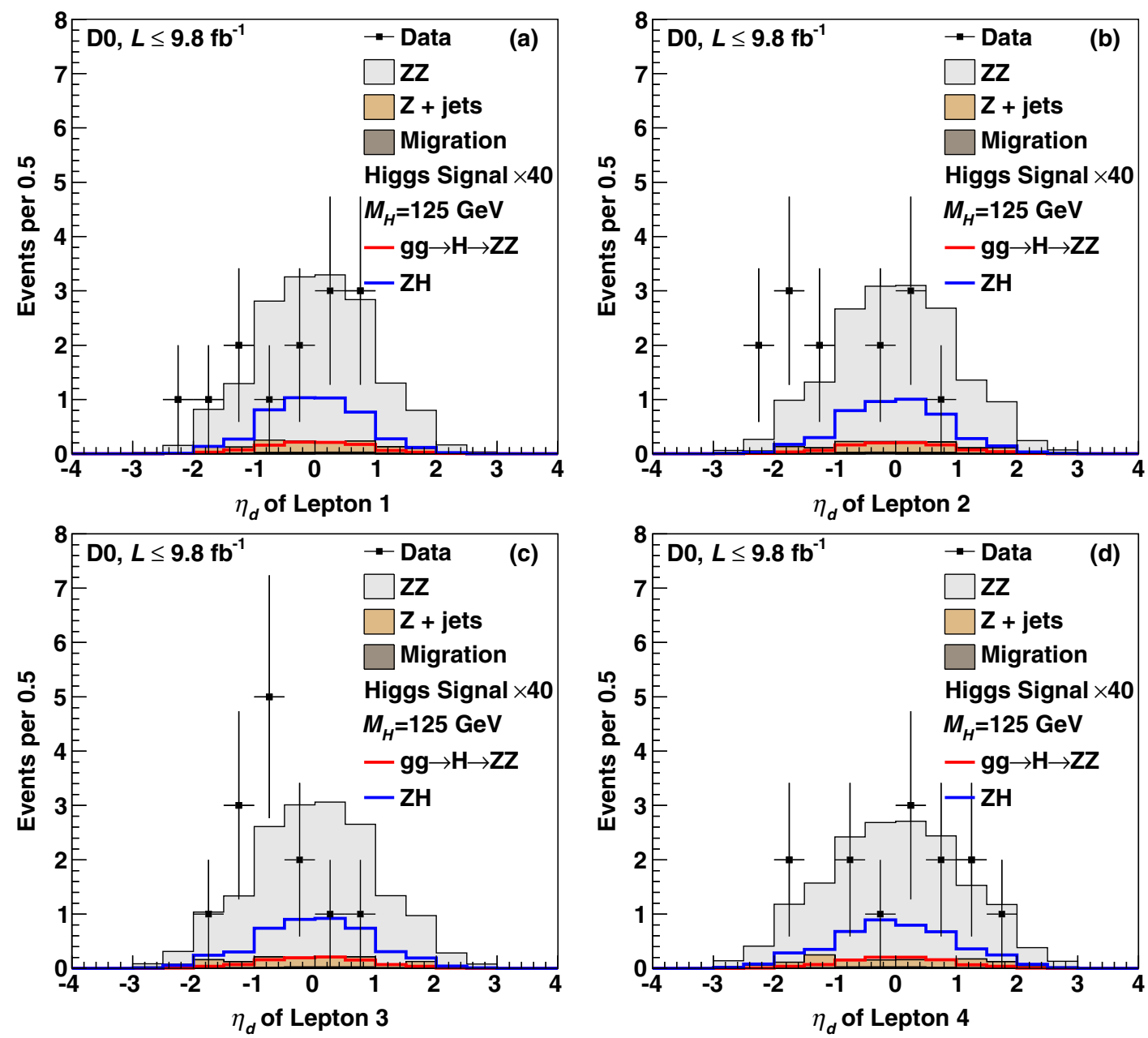

FIG. 6 (color online). Distributions of $\eta_{d}$ in data, expected signal, and backgrounds for the (a) highest- $p_{T}$, (b) second-highest- $p_{T}$, (c) third-highest- $p_{T}$, and (d) lowest- $p_{T}$ leptons in each event. The Higgs boson signal for $M_{H}$ of $125 \mathrm{GeV}$ is shown scaled by a factor of 40 .
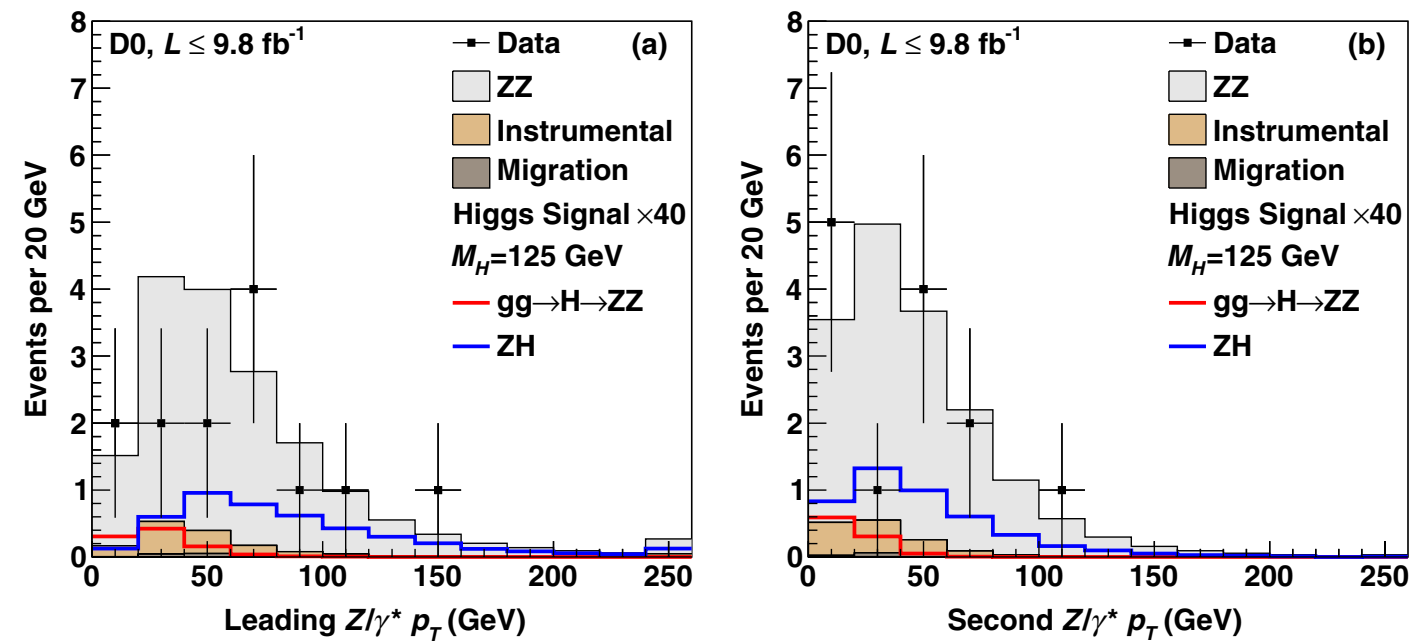

FIG. 7 (color online). Distributions of the $Z / \gamma^{*} p_{T}$ for the (a) leading and (b) second-highest- $p_{T}$ lepton pairings in each event. In the eeee and $\mu \mu \mu \mu$ channels, the combination shown is that with one dilepton mass most consistent with a $Z$ mass of $91.2 \mathrm{GeV}$. The Higgs boson signal for $M_{H}$ of $125 \mathrm{GeV}$ is shown scaled by a factor of 40 . 

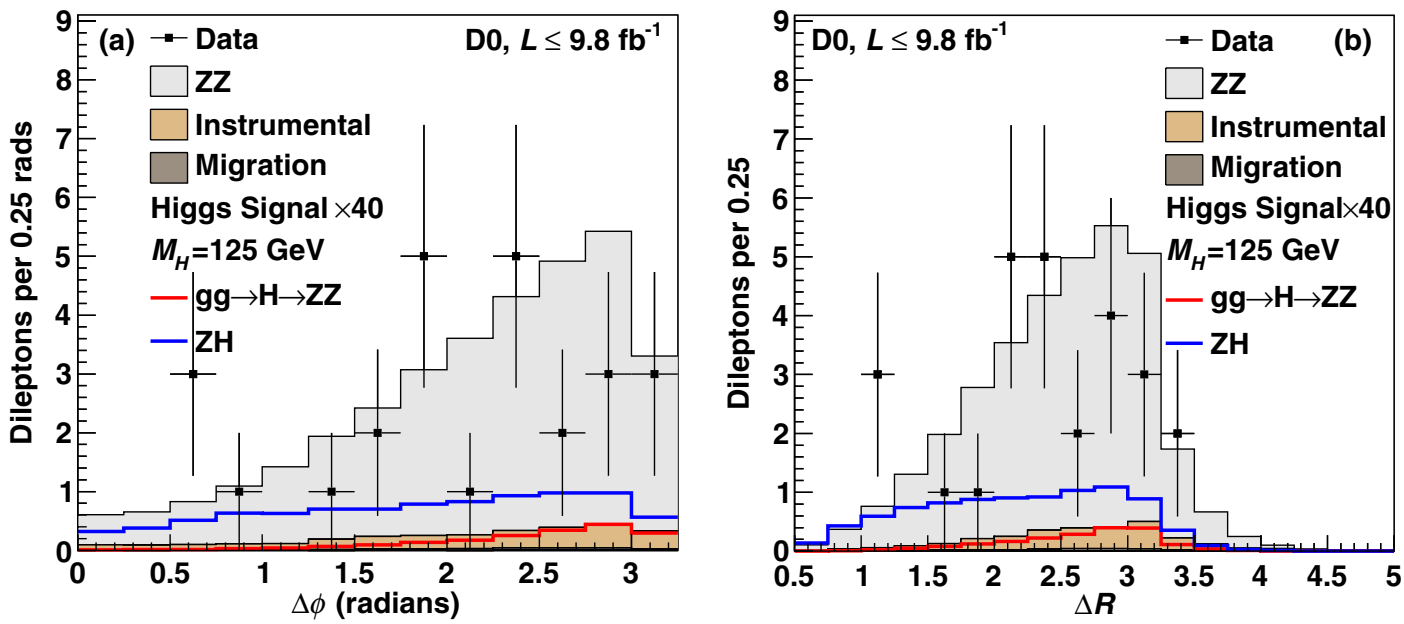

FIG. 8 (color online). Distributions of (a) the opening azimuthal angle, $\Delta \phi$, and (b) the $\Delta R$, between the two leptons of a $Z / \gamma^{*}$. In the eeee and $\mu \mu \mu \mu$ channels, the combination shown is that with one dilepton mass most consistent with a $Z$ mass of $91.2 \mathrm{GeV}$. There are two entries per event in both distributions. The Higgs boson signal for $M_{H}$ of $125 \mathrm{GeV}$ is shown scaled by a factor of 40 .

(a)
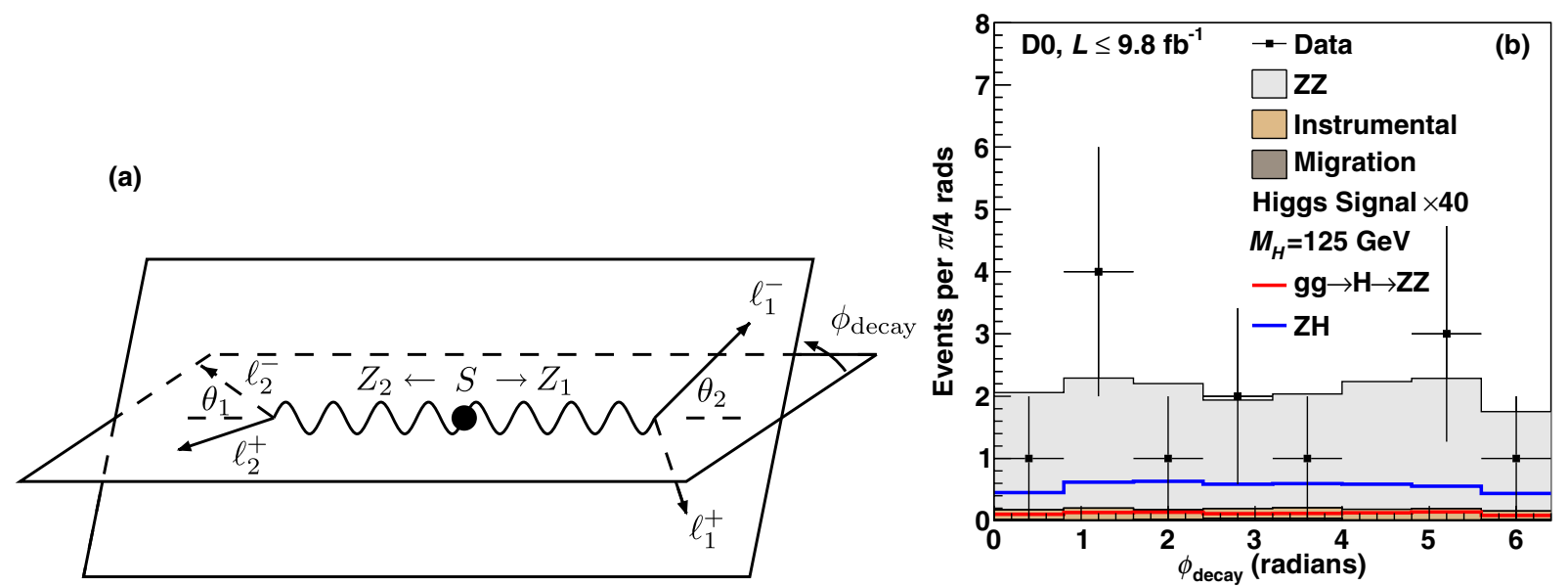

FIG. 9 (color online). Shown in (a) is definition of $\phi_{\text {decay }}$, adapted from Ref. [43] with permission. Shown in (b) is the distribution of the azimuthal $\phi_{\text {decay }}$ angle. In the eeee and $\mu \mu \mu \mu$ channels, $\phi_{\text {decay }}$ is calculated between the combination that is most consistent with a $Z$ mass of $91.2 \mathrm{GeV}$ for one of the two dileptons. The Higgs boson signal for $M_{H}$ of $125 \mathrm{GeV}$ is shown scaled by a factor of 40 .

second lepton pair are in Fig. 7. Figure 8 shows the distributions of the opening angles between the best matched lepton pairs in each event in the azimuthal angle, $\Delta \phi$, and $\Delta R$. Figure 9 shows the angle $\phi_{\text {decay }}$, which is the angle through which the lepton side of one of the $Z / \gamma^{*}$ boson decay planes is rotated into the lepton side of the other $Z / \gamma^{*}$ boson decay plane, and measured in the center-ofmass frame of the $Z / \gamma^{*} Z / \gamma^{*}$ system [43].
[1] G. Aad et al. (ATLAS Collaboration), Phys. Lett. B 716, 1 (2012).

[2] S. Chatrchyan et al. (CMS Collaboration), Phys. Lett. B 716, 30 (2012).

[3] R. Barate et al. (ALEPH Collaboration), Phys. Lett. B 469, 287 (1999).
[4] J. Abdallah et al. (DELPHI Collaboration), Eur. Phys. J. C 30, 447 (2003).

[5] M. Acciarri et al. (L3 Collaboration), Phys. Lett. B 465, 363 (1999).

[6] G. Abbiendi et al. (OPAL Collaboration), Eur. Phys. J. C 32, 303 (2003). 
[7] J. Alcaraz et al. (ALEPH, DELPHI, L3, OPAL, and LEP Electroweak Working Group collaborations), arXiv:hepex/0612034.

[8] V. M. Abazov et al. (D0 Collaboration), Phys. Rev. Lett. 100, 131801 (2008).

[9] V. M. Abazov et al. (D0 Collaboration), Phys. Rev. Lett. 101, 171803 (2008).

[10] V. M. Abazov et al. (D0 Collaboration), Phys. Rev. D 78, 072002 (2008).

[11] V. M. Abazov et al. (D0 Collaboration), Phys. Rev. D 85, 112005 (2012).

[12] T. Aaltonen et al. (CDF Collaboration), Phys. Rev. Lett. 100, 201801 (2008).

[13] G. Aad et al. (ATLAS Collaboration), Phys. Rev. Lett. 108, 041804 (2012).

[14] S. Chatrchyan et al. (CMS Collaboration), J. High Energy Phys. 01 (2013) 063.

[15] S. Chatrchyan et al. (CMS Collaboration), J. High Energy Phys. 12 (2012) 034.

[16] V. M. Abazov et al. (D0 Collaboration), Phys. Rev. D 84, 011103 (2011).

[17] V. M. Abazov et al. (D0 Collaboration), Nucl. Instrum. Methods Phys. Res., Sect. A 565, 463 (2006).

[18] R. Angstadt et al., Nucl. Instrum. Methods Phys. Res., Sect. A 622, 298 (2010).

[19] S. N. Ahmed et al., Nucl. Instrum. Methods Phys. Res., Sect. A 634, 8 (2011).

[20] M. Abolins et al., Nucl. Instrum. Methods Phys. Res., Sect. A 584, 75 (2008).

[21] The pseudorapidity is given by $\eta=-\ln [\tan (\theta / 2)]$, where $\theta$ is the polar angle with respect to the proton beam direction. We define $\eta$ with respect to the $p \bar{p}$ interaction vertex and $\eta_{d}$ with respect to the nominal center of the detector.

[22] V. M. Abazov et al., Nucl. Instrum. Methods Phys. Res., Sect. A 552, 372 (2005).

[23] T. Sjöstrand, S. Mrenna, and P. Skands, J. High Energy Phys. 05 (2006) 026; we use version 6.323.

[24] T. Gleisberg, S. Hoeche, F. Krauss, A. Schaelicke, S. Schumann, and J. Winter, J. High Energy Phys. 02 (2004) 056; , 02 (2009) 007; we use version 1.0.11.
[25] J. M. Campbell and R. K. Ellis, Phys. Rev. D 60, 113006 (1999); we use version 6.2.

[26] J. Pumplin, D. R. Stump, J. Huston, H.-L. Lai, P. Nadolsky, and W.-K. Tung, J. High Energy Phys. 07 (2002) 012.

[27] M. L. Mangano, F. Piccinini, A. D Polosa, M. Moretti, and R. Pittau, J. High Energy Phys. 07 (2003) 001; we use version 2.11.

[28] S. Moch and P. Uwer, Phys. Rev. D 78, 034003 (2008).

[29] D. de Florian and M. Grazzini, Phys. Lett. B 674, 291 (2009).

[30] J. Baglio and A. Djouadi, J. High Energy Phys. 10 (2010) 064; we use version 3.53.

[31] A. Djouadi, J. Kalinowski, and M. Spira, Comput. Phys. Commun. 108, 56 (1998).

[32] R. Brun and F. Carminati, CERN Program Library Long Writeup Report No. W5013, 1993.

[33] The variable $\Delta R$ between two objects $i$ and $j$ is defined as $\Delta R=\sqrt{\left(\eta_{i}-\eta_{j}\right)^{2}+\left(\phi_{i}-\phi_{j}\right)^{2}}$, where $\phi$ is the azimuthal angle.

[34] G. Blazey et al., in Proceedings of Physics at Run II: QCD and Weak Boson Physics Workshop: Final General Meeting, edited by U. Bauer, R. Ellis, and D. Zeppenfeld (Fermilab, Batavia, USA, 2000), p. 47.

[35] V. M. Abazov et al. (D0 Collaboration), Phys. Rev. D 85, 052006 (2012).

[36] The acoplanarity is given by $\phi_{1}-\phi_{2}-\pi$, where $\phi$ is the azimuthal angle.

[37] T. Andeen et al., Report No. FERMILAB-TM-2365, 2007.

[38] O. Brandt et al., Report No. FERMILAB-TM-2540-PPD, 2012.

[39] J. Beringer et al. (Particle Data Group), Phys. Rev. D 86, 010001 (2012).

[40] T. Junk, Nucl. Instrum. Methods Phys. Res., Sect. A 434, 435 (1999).

[41] A. Read, J. Phys. G 28, 2693 (2002).

[42] W. Fisher, Report No. FERMILAB-TM-2386-E, 2006.

[43] Q. Cao, C. B. Jackson, W.-Y. Keung, I. Low, and J. Shu, Phys. Rev. D 81, 015010 (2010). 